\title{
Derivation of a Matrix Product Representation for the Asymmetric Exclusion Process from Algebraic Bethe Ansatz
}

\author{
O. Golinelli, K. Mallick \\ Service de Physique Théorique, Cea Saclay, 91191 Gif, France \\ April 13, 2006 \\ arXiv:cond-mat/0604338
}

\begin{abstract}
We derive, using the algebraic Bethe Ansatz, a generalized Matrix Product Ansatz for the asymmetric exclusion process (ASEP) on a one-dimensional periodic lattice. In this Matrix Product Ansatz, the components of the eigenvectors of the ASEP Markov matrix can be expressed as traces of products of non-commuting operators. We derive the relations between the operators involved and show that they generate a quadratic algebra. Our construction provides explicit finite dimensional representations for the generators of this algebra.
\end{abstract}

Keywords: ASEP, Matrix Product Ansatz, Algebraic Bethe Ansatz.

Pacs number: 05.40.-a, 05.60.-k

\section{Introduction}

The asymmetric simple exclusion process (ASEP) that plays a fundamental role in the theoretical studies of non-equilibrium statistical mechanics, is a driven lattice gas model in which particles interact by hard core exclusion. The ASEP was originally introduced as a building block for models of one dimensional transport where geometric constraints play an important role (e.g., hopping conductivity, motion of RNA templates and traffic flow). 
The exclusion process is a stochastic Markovian model whose dynamical rules are encoded in an evolution (Markov) matrix. Exact results for the ASEP in one dimension have been derived using two complementary approaches, the Matrix Product Ansatz and the Bethe Ansatz (for a review see Derrida 1998, Schütz 2001). The Matrix Product Ansatz (MPA) (Derrida et al. 1993) is based on a representation of the components of the steady state wave function of the Markov operator in terms of a product of matrices. This method has been used to calculate steady state properties of the ASEP such as the invariant measure (Speer 1993), current fluctuations in the stationary state and large deviation functionals (Derrida et al. 2003).

The ASEP is equivalent to a non-hermitian Heisenberg spin chain of the XXZ type and can be mapped into a two-dimensional six-vertex model at equilibrium : these mappings permit the use of integrable systems techniques, such as the Bethe Ansatz. The Bethe Ansatz provides spectral information about the evolution operator (Dhar 1987, Gwa and Spohn 1992; Kim 1995, Golinelli and Mallick 2004) which can then be used to derive large deviation functions (Derrida and Lebowitz 1998).

The exact relation between these two techniques has been a matter of investigation for a long time (Alcaraz et al. 1994; Stinchcombe and Schütz 1995). In a recent work, Alcaraz and Lazo (2004) have expressed the eigenvectors of integrable quantum chains (such as the anisotropic Heisenberg chain) as traces of products of generators of a quadratic algebra. This Matrix Product Ansatz leads to the Bethe Ansatz equations of the system.

In this work, we solve the inverse problem: we prove that a Matrix Product representation involving quadratic algebraic relations between operators can be deduced and constructed explicitly by applying the Algebraic Bethe Ansatz to the ASEP. The quadratic algebra we obtain is similar to the one studied by Alcaraz and Lazo. However, our algebra satisfies different boundary conditions that modify drastically the properties of its representations and ensure the existence of finite dimensional representations.

The outline of this work is as follows. In section 2, we apply the Algebraic Bethe Ansatz to the totally asymmetric exclusion process. In section 3. we derive the Matrix Product Ansatz from the Algebraic Bethe Ansatz. In section 4. we establish the quadratic algebraic relations satisfied by the operators of the MPA. Our results are generalized to the partially asymmetric exclusion process in Section 5. Concluding remarks are presented in the last section. In the appendix, we derive the Bethe Ansatz equations from the quadratic algebra of section 4. 


\section{Algebraic Bethe Ansatz for ASEP}

We consider the exclusion process on a periodic one dimensional lattice with $L$ sites (sites $i$ and $L+i$ are identical). A lattice site cannot be occupied by more than one particle. To represent the state of a site, $i(1 \leq i \leq L)$ we use the spin-1/2 language : a site $i$ can be in two states that we label as $|\uparrow\rangle$ ( $i$ is occupied) and $|\downarrow\rangle$ ( $i$ is empty). A configuration $C$ is represented either by a vector of the type $|\downarrow \uparrow \ldots \uparrow\rangle$ or by a binary vector

$$
|C\rangle=\left|\tau_{1}, \ldots, \tau_{L}\right\rangle \text {. }
$$

where $\tau_{i}=1$ if the site $i$ is occupied and $\tau_{i}=0$ otherwise. The space of all possible configurations is a $2^{L}$ dimensional vector space that we shall denote by $\mathcal{S}$.

The system evolves with time according to the following stochastic rule: a particle on a site $i$ at time $t$ jumps, in the interval between $t$ and $t+d t$, with probability $p d t$ to the neighbouring site $i+1$ if this site is empty (exclusion rule) and with probability $q d t$ to the site $i-1$ if this site is empty. The jump rates $p$ and $q$ are normalized such that $p+q=1$. In the totally asymmetric exclusion process (TASEP), the jumps are totally biased in one direction $(p=1$ and $q=0)$. For sake of simplicity, we shall discuss the TASEP case in full detail. The general case will be considered briefly in Section [5.

We call $\psi_{t}(C)$ the probability of a configuration $C$ at time $t$. As the exclusion process is a continuous-time Markov process, the time evolution of $\psi_{t}(C)$ is determined by the master equation

$$
\frac{d}{d t} \psi_{t}(C)=\sum_{C^{\prime}} M\left(C, C^{\prime}\right) \psi_{t}\left(C^{\prime}\right),
$$

where the element $M\left(C, C^{\prime}\right)$ is the transition rate from configuration $C^{\prime}$ to $C$ and the diagonal term $M(C, C)=-\sum_{C^{\prime}} M\left(C^{\prime}, C\right)$ represents the exit rate from configuration $C$. The Markov matrix $M$, that encodes the dynamics of the exclusion process, is a square matrix of size $2^{L}$ acting on the configuration space $\mathcal{S}$ of the TASEP. This Markov matrix can be expressed as a sum of local operators that update the bond $(i, i+1)$ :

$$
M=\sum_{i=1}^{L} M_{i, i+1},
$$

where the TASEP local update operator $M_{i, i+1}$ is given by

$$
M_{i, i+1}=\mathbf{1}_{1} \otimes \mathbf{1}_{2} \ldots \mathbf{1}_{i-1} \otimes\left(\begin{array}{rrrr}
0 & 0 & 0 & 0 \\
0 & -1 & 0 & 0 \\
0 & 1 & 0 & 0 \\
0 & 0 & 0 & 0
\end{array}\right) \otimes \mathbf{1}_{i+2} \ldots \mathbf{1}_{L}
$$


The matrix $\mathbf{1}_{j}$ is the $2 \times 2$ identity matrix acting on the site $j$ and the $4 \times 4$ matrix appearing in this equation is written in the local basis $(|\uparrow \uparrow\rangle,|\uparrow \downarrow\rangle$, $|\downarrow \uparrow\rangle,|\downarrow \downarrow\rangle)$, that represents the four possible states of the bond $(i, i+1)$. Thus, $M_{i, i+1}$ is a $2^{L}$ matrix that acts trivially on all sites other than $i$ and $i+1$ and makes a particle jump from site $i$ to the site $i+1$.

It is also useful to define the permutation operator $P_{i, i+1}$ that exchanges the states of sites $i$ and $i+1$ :

$$
P_{i, i+1}=\mathbf{1}_{1} \otimes \mathbf{1}_{2} \ldots \mathbf{1}_{i-1} \otimes\left(\begin{array}{cccc}
1 & 0 & 0 & 0 \\
0 & 0 & 1 & 0 \\
0 & 1 & 0 & 0 \\
0 & 0 & 0 & 1
\end{array}\right) \otimes \mathbf{1}_{i+2} \ldots \mathbf{1}_{L}
$$

More generally, we can define a jump operator $M_{i, j}$ and a permutation operator $P_{i, j}$, between sites $i$ and $j$ that act trivially on all sites other than $i$ and $j ; M_{i, j}$ makes a particle jump from site $i$ to site $j$ and $P_{i, j}$ exchanges the states of sites $i$ and $j$.

In the Algebraic Bethe Ansatz method (see e.g., Nepomechie, 1999 for an introduction to this subject), an auxiliary site $a$ is introduced, which can be in two states labelled as $|1\rangle$ (site $a$ is occupied) and $|2\rangle$ (site $a$ is empty). These two states span a two dimensional vector space, $\mathcal{A}$, the auxiliary space. We now define an operator $\mathcal{L}_{i}(\lambda)$ that acts on the tensor space $\mathcal{A} \otimes \mathcal{S}$; this operator acts trivially on all sites other than $a$ and $i$, and is a function of a spectral parameter $\lambda$

$$
\mathcal{L}_{i}(\lambda)=P_{i, a}\left(1+\lambda M_{i, a}\right) .
$$

This operator can also be represented as a $2 \times 2$ operator on the vector space $\mathcal{A}$

$$
\mathcal{L}_{i}(\lambda)=\left(\begin{array}{ll}
a(\lambda) & b(\lambda) \\
c(\lambda) & d(\lambda)
\end{array}\right),
$$

where the matrix elements $a(\lambda), b(\lambda), c(\lambda)$ and $d(\lambda)$ are themselves $2^{L} \times 2^{L}$ operators that act on the configuration space $\mathcal{S}$. These operators act trivially on all sites different from $i$ and are given by

$$
\begin{aligned}
& a(\lambda)=\mathbf{1}_{1} \otimes \ldots \mathbf{1}_{i-1} \otimes\left(\begin{array}{cc}
1 & 0 \\
0 & 0
\end{array}\right) \otimes \mathbf{1}_{i+1} \ldots \mathbf{1}_{L}, \\
& b(\lambda)=\mathbf{1}_{1} \otimes \ldots \mathbf{1}_{i-1} \otimes\left(\begin{array}{cc}
0 & 0 \\
1-\lambda & 0
\end{array}\right) \otimes \mathbf{1}_{i+1} \ldots \mathbf{1}_{L}, \\
& c(\lambda)=\mathbf{1}_{1} \otimes \ldots \mathbf{1}_{i-1} \otimes\left(\begin{array}{cc}
0 & 1 \\
0 & 0
\end{array}\right) \otimes \mathbf{1}_{i+1} \ldots \mathbf{1}_{L}, \\
& d(\lambda)=\mathbf{1}_{1} \otimes \ldots \mathbf{1}_{i-1} \otimes\left(\begin{array}{cc}
\lambda & 0 \\
0 & 1
\end{array}\right) \otimes \mathbf{1}_{i+1} \ldots \mathbf{1}_{L} .
\end{aligned}
$$


The tensor products in these equations represent products over the twodimensional local configuration space of a site.

The operators $\mathcal{L}_{i}(\lambda)$ satisfy a Yang-Baxter type relation. We consider the operator

$$
\mathcal{R}(\nu)=1+\nu M_{a^{\prime}, a},
$$

that acts on $\mathcal{A} \otimes \mathcal{A}^{\prime}$ where the auxiliary spaces $\mathcal{A}$ and $\mathcal{A}^{\prime}$ correspond to the auxiliary sites $a$ and $a^{\prime}$. In the basis $\left(\left|1_{a}, 1_{a^{\prime}}\right\rangle,\left|1_{a}, 2_{a^{\prime}}\right\rangle,\left|2_{a}, 1_{a^{\prime}}\right\rangle,\left|2_{a}, 2_{a^{\prime}}\right\rangle\right)$ of $\mathcal{A} \otimes \mathcal{A}^{\prime}$, the operator $\mathcal{R}(\nu)$ is represented by a $4 \times 4$ scalar matrix :

$$
\mathcal{R}(\nu)=\left(\begin{array}{cccc}
1 & 0 & 0 & 0 \\
0 & 1 & \nu & 0 \\
0 & 0 & 1-\nu & 0 \\
0 & 0 & 0 & 1
\end{array}\right)
$$

The following identity is then satisfied

$$
\mathcal{R}(\nu)\left[\mathcal{L}_{i}(\lambda) \otimes \mathcal{L}^{\prime}{ }_{i}(\mu)\right]=\left[\mathcal{L}_{i}(\mu) \otimes \mathcal{L}^{\prime}{ }_{i}(\lambda)\right] \mathcal{R}(\nu) \text { with } \nu=\frac{\lambda-\mu}{1-\mu},
$$

where $\mathcal{L}_{i}$ and $\mathcal{L}^{\prime}{ }_{i}$ are interpreted as $2 \times 2$ matrices acting on $\mathcal{A}$ and $\mathcal{A}^{\prime}$, respectively, with matrix elements that are themselves operators on $\mathcal{S}$. Their tensor product is thus a $4 \times 4$ matrix, acting on $\mathcal{A} \otimes \mathcal{A}^{\prime}$ with matrix elements that are operators on $\mathcal{S}$.

The monodromy matrix is defined as

$$
T(\lambda)=\mathcal{L}_{1}(\lambda) \mathcal{L}_{2}(\lambda) \ldots \mathcal{L}_{L}(\lambda)
$$

where the product of the $\mathcal{L}_{i}$ 's has to be understood as a product of $2 \times$ 2 matrices acting on $\mathcal{A}$ with non-commutative elements. The monodromy matrix $T(\lambda)$ can thus be written as

$$
T(\lambda)=\left(\begin{array}{ll}
A(\lambda) & B(\lambda) \\
C(\lambda) & D(\lambda)
\end{array}\right)
$$

where $A, B, C$ and $D$ are operators on the configuration space $\mathcal{S}$. Taking the trace of the monodromy matrix over the auxiliary space $\mathcal{A}$, we obtain a one-parameter family of transfer matrices acting on $\mathcal{S}$

$$
t(\lambda)=\operatorname{Tr}_{\mathcal{A}}(T(\lambda))=A(\lambda)+D(\lambda)
$$

Equation (14) implies that the operators $t(\lambda)$ form a family of commuting operators (see e.g., Nepomechie, 1999). In particular, this family contains the translation operator $\mathcal{T}=t(0)$ (that shifts all the particles simultaneously one 
site forward) and the Markov matrix $M=t^{\prime}(0) / t(0)$. Using Algebraic Bethe Ansatz, the common eigenvectors of this family are explicitly constructed by actions of the $B$ operators on the reference state $\Omega$, defined as

$$
\Omega=|\uparrow \uparrow \ldots \uparrow\rangle .
$$

The state $\Omega$ corresponds to a configuration where all the sites are occupied.

More precisely, for any $n \leq L$, we define the vector

$$
\left|z_{1}, z_{2} \ldots z_{n}\right\rangle=B\left(z_{n}\right) \ldots B\left(z_{2}\right) B\left(z_{1}\right) \Omega
$$

where $z_{1}, z_{2} \ldots z_{n}$ are complex numbers. Because each operator $B$ creates a hole in the system, the state $\left|z_{1}, z_{2} \ldots z_{n}\right\rangle$ is a linear combination of configurations with exactly $n$ holes. This vector is an eigenvector of the operator $t(\lambda)$ (for all values of $\lambda$ ) and in particular of the Markov matrix M, provided the pseudo-moments $z_{1}, z_{2}, \ldots, z_{n}$ satisfy the Bethe equations :

$$
z_{l}^{L}=(-1)^{n-1} \prod_{i=1}^{n} \frac{1-z_{l}}{1-z_{i}} \text { for } l=1 \ldots n .
$$

The corresponding eigenvalue of $t(\lambda)$ is given by

$$
E(\lambda)=\frac{(1-\lambda)^{n}+\lambda^{L} \prod_{i=1}^{n}\left(z_{i}-1\right)}{\prod_{i=1}^{n}\left(z_{i}-\lambda\right)} .
$$

Using the Bethe equations (20), we find that $E(\lambda)$ is a polynomial in $\lambda$ of degree $L-n$.

\section{Derivation of the Matrix Product Repre- sentation from Bethe Ansatz}

In the previous section, we have constructed the eigenvectors of the Markov matrix by using the the Algebraic Bethe Ansatz, see equation (19). In this section we show that the Algebraic Bethe Ansatz also permits us to express the components of an eigenvector as a Matrix Product (Mallick 1996). From equation (16), we remark that

$$
B(\lambda)=\langle 1|T(\lambda)| 2\rangle .
$$

Using this relation, the eigenvector given in equation (19) can be written as

$$
\begin{aligned}
\left|z_{1}, z_{2} \ldots z_{n}\right\rangle & =\left\langle 1\left|T\left(z_{n}\right)\right| 2\right\rangle \ldots\left\langle 1\left|T\left(z_{2}\right)\right| 2\right\rangle\left\langle 1\left|T\left(z_{1}\right)\right| 2\right\rangle \Omega \\
& =\left\langle 1,1, \ldots, 1\left|T\left(z_{n}\right) \ldots \otimes T\left(z_{2}\right) \otimes T\left(z_{1}\right)\right| 2,2, \ldots, 2\right\rangle \Omega \\
& =\operatorname{Tr}\left(Q_{n} T\left(z_{n}\right) \otimes \ldots \otimes T\left(z_{1}\right) \Omega\right),
\end{aligned}
$$


where the tensor-products act on the space $\mathcal{A}^{\otimes n}$ with $n$ auxiliary sites. The boundary operator $Q_{n}$ is given by

$$
Q_{n}=|2,2, \ldots, 2\rangle\langle 1,1, \ldots, 1| .
$$

Using the definition (16) of the $T$ matrix, we rewrite equation (23) as follows

$$
\begin{aligned}
\left|z_{1}, z_{2} \ldots, z_{n}\right\rangle & =\operatorname{Tr}\left(Q_{n} \prod_{i=1}^{L} \mathcal{L}_{i}\left(z_{1}, \ldots, z_{n}\right) \Omega\right), \\
\text { with } \quad \mathcal{L}_{i}\left(z_{1}, \ldots, z_{n}\right) & =\mathcal{L}_{i}\left(z_{n}\right) \otimes \ldots \otimes \mathcal{L}_{i}\left(z_{1}\right) .
\end{aligned}
$$

The operator $\mathcal{L}_{i}\left(z_{1}, \ldots, z_{n}\right)$ is a $2^{n} \times 2^{n}$ matrix acting on $\mathcal{A}^{\otimes n}$. The matrix elements of $\mathcal{L}_{i}\left(z_{1}, \ldots, z_{n}\right)$ are operators on the configuration space $\mathcal{S}$ that act trivially on all sites except the site $i$. We now define the two operators $D_{n}$ and $E_{n}$ by the following relation

$$
\mathcal{L}_{i}\left(z_{1}, \ldots, z_{n}\right)|\uparrow\rangle=D_{n}\left(z_{1}, \ldots, z_{n}\right)|\uparrow\rangle+E_{n}\left(z_{1}, \ldots, z_{n}\right)|\downarrow\rangle,
$$

where $|\uparrow\rangle$ and $|\downarrow\rangle$ represent the states of the site $i$. (For sake of simplicity, we are writing $|\uparrow\rangle$ and $|\downarrow\rangle$ instead of $\left|\uparrow_{i}\right\rangle$ and $\left.\left|\downarrow_{i}\right\rangle\right)$.

Equivalently, these two operators are given by

$$
\begin{aligned}
& D_{n}\left(z_{1}, \ldots, z_{n}\right)=\left\langle\uparrow\left|\mathcal{L}_{i}\left(z_{1}, \ldots, z_{n}\right)\right| \uparrow\right\rangle, \\
& E_{n}\left(z_{1}, \ldots, z_{n}\right)=\left\langle\downarrow\left|\mathcal{L}_{i}\left(z_{1}, \ldots, z_{n}\right)\right| \uparrow\right\rangle .
\end{aligned}
$$

The operators $D_{n}, E_{n}$ and $Q_{n}$ are $2^{n} \times 2^{n}$ matrices acting on $\mathcal{A}^{\otimes n}$ with scalar elements. We shall now prove some recursion relations satisfied by $D_{n}, E_{n}$ and $Q_{n}$. For $n=1$, we have

$$
D_{1}\left(z_{1}\right)=\left(\begin{array}{cc}
1 & 0 \\
0 & z_{1}
\end{array}\right), E_{1}\left(z_{1}\right)=\left(\begin{array}{cc}
0 & 1-z_{1} \\
0 & 0
\end{array}\right) \text { and } Q_{1}=\left(\begin{array}{ll}
0 & 0 \\
1 & 0
\end{array}\right) \text {. }
$$

From equations (26) and (28) we obtain:

$$
D_{n+1}\left(z_{1}, \ldots, z_{n+1}\right)=\left\langle\uparrow\left|\mathcal{L}_{i}\left(z_{n+1}\right) \otimes \mathcal{L}_{i}\left(z_{1}, \ldots, z_{n}\right)\right| \uparrow\right\rangle .
$$

Using the following identities (deduced from equations (811])

$$
\begin{aligned}
\langle\uparrow| a\left(z_{n+1}\right) & =\langle\uparrow|, & & \langle\uparrow| b\left(z_{n+1}\right)=0, \\
\langle\uparrow| c\left(z_{n+1}\right) & =\langle\downarrow|, & & \langle\uparrow| d\left(z_{n+1}\right)=z_{n+1}\langle\uparrow|,
\end{aligned}
$$

we derive the following recursion relation :

$$
\begin{aligned}
D_{n+1}\left(z_{1}, \ldots, z_{n+1}\right) & =\left(\begin{array}{cc}
1 & 0 \\
0 & z_{n+1}
\end{array}\right) \otimes D_{n}+\left(\begin{array}{cc}
0 & 0 \\
1 & 0
\end{array}\right) \otimes E_{n} \\
& =\left(\begin{array}{cc}
D_{n}\left(z_{1}, \ldots, z_{n}\right) & 0 \\
E_{n}\left(z_{1}, \ldots, z_{n}\right) & z_{n+1} D_{n}\left(z_{1}, \ldots, z_{n}\right)
\end{array}\right) .
\end{aligned}
$$


Thus, $D_{n+1}$ is a $2^{n+1} \times 2^{n+1}$ matrix written as a $2 \times 2$ matrix built of blocks of size $2^{n} \times 2^{n}$. Similarly, we have

$$
\begin{aligned}
E_{n+1}\left(z_{1}, \ldots, z_{n+1}\right) & =\left(\begin{array}{cc}
0 & 1-z_{n+1} \\
0 & 0
\end{array}\right) \otimes D_{n}+\left(\begin{array}{ll}
0 & 0 \\
0 & 1
\end{array}\right) \otimes E_{n} \\
& =\left(\begin{array}{cc}
0 & \left(1-z_{n+1}\right) D_{n}\left(z_{1}, \ldots, z_{n}\right) \\
0 & E_{n}\left(z_{1}, \ldots, z_{n}\right)
\end{array}\right) .
\end{aligned}
$$

These recursion relations, together with equation (30), allow to calculate $D_{n}$ and $E_{n}$ for all $n$. In particular, we remark that $D_{n}$ is a lower triangular matrix and its $2^{n}$ eigenvalues are given by $\prod_{k=1}^{n} z_{k}^{\epsilon_{k}}$, with $\epsilon_{k}=0$ or 1 .

Finally, using equation (24), we obtain

$$
Q_{n+1}=\left(\begin{array}{cc}
0 & 0 \\
Q_{n} & 0
\end{array}\right)
$$

We now prove that the operators $D_{n}, E_{n}$ and $Q_{n}$ provide a Matrix Product Representation for the components of the eigenvector $\left|z_{1}, \ldots, z_{n}\right\rangle$ of the Markov matrix. Using equation (11), the components of $\left|z_{1}, \ldots, z_{n}\right\rangle$ on a configuration $C$ of the system can be written as,

$$
\left\langle C \mid z_{1}, \ldots, z_{n}\right\rangle=\operatorname{Tr}\left(Q_{n} \prod_{i=1}^{L}\left(\tau_{i} D_{n}+\left(1-\tau_{i}\right) E_{n}\right)\right),
$$

where $\tau_{i}=1$ if $i$ is occupied in configuration $C$ and $\tau_{i}=0$ otherwise. Hence, a particle is represented by the matrix $D_{n}$ and a hole is represented by the matrix $E_{n}$.

Because of the conservation of the number of particles and holes, the right hand side of equation (39) vanishes when the configuration $C$ does not have exactly $n$ holes. If we call $x_{1}, x_{2}, \ldots, x_{n}$ the positions of the $n$ holes in $C$, equation (39) can be rewritten as

$$
\left\langle x_{1}, \ldots, x_{n} \mid z_{1}, \ldots, z_{n}\right\rangle=\operatorname{Tr}\left(Q_{n} D_{n}^{x_{1}-1} E_{n} D_{n}^{x_{2}-x_{1}-1} E_{n} \ldots D_{n}^{x_{n}-x_{n-1}-1} E_{n} D_{n}^{L-x_{n}}\right)
$$

This expression gives a Matrix Product Representation for any eigenvector of the Markov matrix. This expression generalizes the steady-state Matrix Product introduced by Derrida et al. (1993). In the Appendix, we show that the expression (40) can be recast in the familiar coordinate Bethe Ansatz form. 


\section{Identification of the Quadratic Algebra}

In the previous section, we have derived the Matrix Product Representation from the Algebraic Bethe Ansatz by constructing explicitly the operators $D_{n}$ and $E_{n}$. We now prove that these operators satisfy some simple algebraic relations; more precisely, the operator $E_{n}$ can be decomposed as a sum of $n$ operators $E_{n}^{(i)}$

$$
E_{n}=\sum_{i=1}^{n} E_{n}^{(i)},
$$

that obey the quadratic relations

$$
\begin{aligned}
E_{n}^{(i)} D_{n} & =z_{i} D_{n} E_{n}^{(i)} \\
\text { for } i \neq j:\left(1-\frac{1}{z_{i}}\right) E_{n}^{(i)} E_{n}^{(j)} & =-\left(1-\frac{1}{z_{j}}\right) E_{n}^{(j)} E_{n}^{(i)}, \\
\text { and } E_{n}^{(i)} E_{n}^{(i)} & =0 .
\end{aligned}
$$

In equations (42 44), the scalars $z_{i}$ are arbitrary complex numbers and do not have to be solutions of the Bethe equations. Such a quadratic algebra was postulated by Alcaraz and Lazo (2004) as an Ansatz to diagonalize the Hamiltonian of quantum spin chains. We show here that, for the ASEP, this quadratic algebra can be rigorously deduced from the Algebraic Bethe Ansatz. Our construction is explicit and provides finite dimensional representations of the abstract quadratic algebra defined by relations (42, 44).

We shall prove the relations (41,44) by induction on $n$, by diagonalizing the matrix $D_{n}$ and calculating $E_{n}$ in the new basis. We recall that all the matrices with index $n$ are of size $2^{n} \times 2^{n}$.

For $n=1$, the matrix $D_{1}$ is diagonal, and $D_{1}$ and $E_{1}$ satisfy the relations (41 44). Now, we suppose, by the recursion hypothesis, that we have already diagonalized the matrix $D_{n}$, i.e., we have found an invertible matrix $R_{n}$ such that

$$
R_{n}^{-1} D_{n} R_{n}=\Delta_{n}
$$

where $\Delta_{n}$ is a diagonal matrix with diagonal

$$
\operatorname{diag}\left(\Delta_{n}\right)=\left(1, z_{1}, z_{2}, z_{2} z_{1}, z_{3}, z_{3} z_{1}, z_{3} z_{2}, z_{3} z_{2} z_{1}, \ldots, z_{n} \ldots z_{1}\right) .
$$

(For $n=1$, we have $\Delta_{1}=D_{1}$ and $R_{1}=1$ ). In the new basis, the matrix $E_{n}$ becomes

$$
\mathcal{E}_{n}=R_{n}^{-1} E_{n} R_{n} .
$$

We suppose, again by the recursion hypothesis, that we have found a decomposition of $\mathcal{E}_{n}$

$$
\mathcal{E}_{n}=\sum_{i=1}^{n} \mathcal{E}_{n}^{(i)}
$$


such that the relations (42 44) are satisfied between $\Delta_{n}$ and the $\mathcal{E}_{n}^{(i)}$ 's. Then, by a change of basis, the same relations are also satisfied by $D_{n}=R_{n} \Delta_{n} R_{n}^{-1}$ and $E_{n}^{(i)}=R_{n} \mathcal{E}_{n}^{(i)} R_{n}^{-1}$.

We now show that a similar decomposition can be found at the level $n+1$. We first construct the matrix $R_{n+1}$ that transforms $D_{n+1}$ into the diagonal form (46). Using equation (35), we take $R_{n+1}$ to be of the form

$$
R_{n+1}=\left(\begin{array}{cc}
R_{n} & 0 \\
R_{n} A_{n} & R_{n}
\end{array}\right)
$$

where $A_{n}$ is an unknown matrix to be determined. From equations (35, 45, 47) and 49), we obtain

$$
R_{n+1}^{-1} D_{n+1} R_{n+1}=\left(\begin{array}{cc}
\Delta_{n} & 0 \\
-A_{n} \Delta_{n}+z_{n+1} \Delta_{n} A_{n}+\mathcal{E}_{n} & z_{n+1} \Delta_{n}
\end{array}\right) .
$$

This matrix is diagonal if and only if $A_{n}$ satisfies the relation

$$
A_{n} \Delta_{n}-z_{n+1} \Delta_{n} A_{n}=\mathcal{E}_{n} .
$$

Knowing that $\Delta_{n}$ and $\mathcal{E}_{n}^{(i)}$ satisfy the relations (42 44), we find the solution $A_{n}$ of equation (51):

$$
A_{n}=\Delta_{n}^{-1} \sum_{i=1}^{n} \frac{\mathcal{E}_{n}^{(i)}}{z_{i}-z_{n+1}}
$$

We thus obtain

$$
\Delta_{n+1}=R_{n+1}^{-1} D_{n+1} R_{n+1}=\left(\begin{array}{cc}
\Delta_{n} & 0 \\
0 & z_{n+1} \Delta_{n}
\end{array}\right) ;
$$

$\Delta_{n+1}$ is a diagonal matrix.

The operator $E_{n+1}$ in the new basis is found to be :

$\mathcal{E}_{n+1}=R_{n+1}^{-1} E_{n+1} R_{n+1}=\left(\begin{array}{cc}\left(1-z_{n+1}\right) \sum_{i=1}^{n} \frac{\mathcal{E}_{n}^{(i)}}{z_{i}-z_{n+1}} & \left(1-z_{n+1}\right) \Delta_{n} \\ 0 & z_{n+1} \sum_{i=1}^{n} \frac{\left(z_{i}-1\right) \mathcal{E}_{n}^{(i)}}{z_{i}-z_{n+1}}\end{array}\right)$.

This equation is derived by using equation (52) and the relations (42 44). We emphasize that $\mathcal{E}_{n+1}$ is a strictly upper-triangular matrix : its lower-left elements and its diagonal vanish identically. From equation (54), we deduce the decomposition of $\mathcal{E}_{n+1}$

$$
\mathcal{E}_{n+1}=\sum_{i=1}^{n+1} \mathcal{E}_{n+1}^{(i)}
$$


where

$$
\text { for } \begin{aligned}
i \leq n, \mathcal{E}_{n+1}^{(i)} & =\frac{1}{z_{i}-z_{n+1}}\left(\begin{array}{cc}
\left(1-z_{n+1}\right) \mathcal{E}_{n}^{(i)} & 0 \\
0 & z_{n+1}\left(z_{i}-1\right) \mathcal{E}_{n}^{(i)}
\end{array}\right) \\
\text { and } \mathcal{E}_{n+1}^{(n+1)} & =\left(\begin{array}{cc}
0 & \left(1-z_{n+1}\right) \Delta_{n} \\
0 & 0
\end{array}\right) .
\end{aligned}
$$

Knowing that $\Delta_{n}$ and $\mathcal{E}_{n}^{(i)}$ satisfy the relations (42 44), and using the explicit expressions (53] [56] and [57), we find that the operators $\Delta_{n+1}$ and $\mathcal{E}_{n+1}^{(i)}$ also satisfy the algebraic rules (42 44) for $1 \leq i \leq n+1$. Reverting to the original basis by using the matrix $R_{n+1}$, we conclude that $D_{n+1}$ and $E_{n+1}$ satisfy the same relations. We have thus shown the existence of the quadratic algebraic relations (42 44) at the level $n+1$.

We finally discuss the existence of relations between the operators $D_{n}$ and $E_{n}$, and the boundary operator $Q_{n}$. Using the equations (24) and (35), we obtain the following relation between $D_{n}$ and $Q_{n}$ :

$$
D_{n} Q_{n}=\left(\prod_{i=1}^{n} z_{i}\right) Q_{n} D_{n}=\left(\prod_{i=1}^{n} z_{i}\right) Q_{n} .
$$

We emphasize that in the quadratic algebra that we have derived from the algebraic Bethe Ansatz, there is no algebraic relation between $E_{n} Q_{n}$ and $Q_{n} E_{n}$. Therefore, the quadratic algebra that we have constructed is akin to but not identical to that studied by Alcaraz and Lazo (2004). However, any modification of the boundary relations alters the properties of the algebra and profoundly modifies its representation theory. For example, it can be proved (Golinelli and Mallick, in preparation) that, for the ASEP, the algebra defined in (Alcaraz and Lazo 2004) is such that all its finite dimensional representations have vanishing traces and, therefore, can not be used to construct a Matrix Product Ansatz. In contrast, the algebra we have constructed here admits finite dimensional representations with non-zero trace and therefore allows us to define a bona-fide Matrix Product Ansatz. Besides, from a physical point of view, it is well known that boundary conditions play a crucial role in the ASEP (see e.g., Schütz 2001).

The algebra (42 44) and the boundary equation (58) encode the Bethe Ansatz. In the Appendix, we use these relations to prove ab initio that the vector $\left|z_{1}, \ldots, z_{n}\right\rangle$ whose components are given in equation (40) is an eigenvector of the Markov matrix $M$ provided the pseudo-moments $z_{1}, z_{2}$, $\ldots, z_{n}$ satisfy the Bethe equations (20). 


\section{Generalization}

In this section, we briefly indicate how to generalize our results to the partially asymmetric exclusion process in which the particles hop to the right and to the left with jump rates given by $p$ and $q$ respectively. For the partially asymmetric exclusion process the local update operator $M_{i, i+1}$ is given by (we omit the identity operators for sake of clarity) :

$$
M_{i, i+1}=\left(\begin{array}{cccc}
0 & 0 & 0 & 0 \\
0 & -p & q & 0 \\
0 & p & -q & 0 \\
0 & 0 & 0 & 0
\end{array}\right) \text {. }
$$

Using this local operator, the construction of the $\mathcal{L}_{i}$ matrices and the monodromy operator $T$ is identical to that explained above. The recursion relations (35) and (37) for the $D_{n}$ and $E_{n}$ operators, respectively, are now replaced by :

$$
D_{n+1}\left(z_{1}, \ldots, z_{n+1}\right)=\left(\begin{array}{cc}
D_{n}\left(z_{1}, \ldots, z_{n}\right) & 0 \\
\left(1-\frac{q}{p} z_{n+1}\right) E_{n}\left(z_{1}, \ldots, z_{n}\right) & z_{n+1} D_{n}\left(z_{1}, \ldots, z_{n}\right)
\end{array}\right)
$$

and

$$
E_{n+1}\left(z_{1}, \ldots, z_{n+1}\right)=\left(\begin{array}{cc}
\frac{q}{p} z_{n+1} E_{n}\left(z_{1}, \ldots, z_{n}\right) & \left(1-z_{n+1}\right) D_{n}\left(z_{1}, \ldots, z_{n}\right) \\
0 & E_{n}\left(z_{1}, \ldots, z_{n}\right)
\end{array}\right)
$$

The operators $D_{1}$ and $E_{1}$ are identical to those defined in equation (30).

Here also, $E_{n}$ can be written as a sum of $n$ operators $E_{n}^{(i)}$ as in equation (41). The operators $E_{n}^{(i)}$ and $D_{n}$ generate a quadratic algebra. The relations (42) and (44) still hold good but the equation (43) is replaced by

$$
\text { for } i \neq j:\left(1-\frac{p}{z_{i}}-q z_{j}\right) E_{n}^{(i)} E_{n}^{(j)}=-\left(1-\frac{p}{z_{j}}-q z_{i}\right) E_{n}^{(j)} E_{n}^{(i)} .
$$

These algebraic relation are obtained, again, by recursion on $n$. The diagonal basis is found by using the transformation $R_{n}$ defined recursively in equation (49) where the matrix $A_{n}$ is now given by

$$
A_{n}=\left(1-\frac{q}{p} z_{n+1}\right) \Delta_{n}^{-1} \sum_{i=1}^{n} \frac{\mathcal{E}_{n}^{(i)}}{z_{i}-z_{n+1}}
$$

For $q=0$, we recover the expressions given in sections 3 and 4 


\section{Conclusion}

In this work, we have shown that the components of the eigenvectors of the asymmetric exclusion process can be written as traces over matrix products. This Matrix Product Representation has been constructed from the Algebraic Bethe Ansatz in a systematic manner (40). Our method also allows to derive the algebraic relations (41-44) satisfied by the operators that represent particles and holes. The quadratic relations obtained are in fact logical consequences of the Algebraic Bethe Ansatz procedure and thus, ultimately, stem from the Yang-Baxter equation. The approach described in this work shows that there is a close relation between the Matrix method and the Bethe Ansatz, at least in the case of the ASEP. We believe that the derivation of the Matrix Ansatz for the ASEP presented here can be generalized to integrable quantum chains. Besides, if the equivalence between Matrix Ansatz and Bethe Ansatz is true in general, this would provide a technique for constructing the quadratic algebras from first principles rather that having to postulate them a priori. We also emphasize that, in contrast with the work of Alcaraz and Lazo (2004), our construction provides explicit finite dimensional representations of the algebras involved that can be used for actual calculations on finite size systems.

\section{Acknowledgments}

We thank Bernard Derrida, Vincent Hakim and Vincent Pasquier for help and encouragement at early stages of this work. We also thank S. Mallick for a careful reading of the manuscript.

\section{Appendix: Derivation of the Bethe Equations}

We explain here how to derive the Bethe equations (20) from the quadratic algebra (42 44) and the boundary relation (58). We first show that the expression (40) is equivalent to the standard coordinate Bethe Ansatz form:

$$
\begin{aligned}
& \left\langle x_{1}, \ldots, x_{n} \mid z_{1}, \ldots, z_{n}\right\rangle= \\
& \operatorname{Tr}\left(Q_{n} D_{n}^{x_{1}-1} E_{n} D_{n}^{x_{2}-x_{1}-1} E_{n} \ldots D_{n}^{x_{n}-x_{n-1}-1} E_{n} D_{n}^{L-x_{n}}\right)= \\
& \sum_{\sigma \in \Sigma_{n}} \operatorname{Tr}\left(Q_{n} D_{n}^{x_{1}-1} E_{n}^{(\sigma(1))} D_{n}^{x_{2}-x_{1}-1} E_{n}^{(\sigma(2))} \ldots D_{n}^{x_{n}-x_{n-1}-1} E_{n}^{(\sigma(n))} D_{n}^{L-x_{n}}\right)(64
\end{aligned}
$$

where $\sigma$ belongs to $\Sigma_{n}$ the permutation group of $n$ objects. This formula is obtained by inserting the decomposition (41) for the operators $E_{n}$ and by 
noticing from relation (44) that each $E_{n}^{(i)}$ must appear only once. We use equation (42) to push all the operators $E_{n}^{(\sigma(i))}$ to the right. We thus obtain

$$
\begin{aligned}
& \left\langle x_{1}, \ldots, x_{n} \mid z_{1}, \ldots, z_{n}\right\rangle= \\
& \sum_{\sigma \in \Sigma_{n}} z_{\sigma(n)}^{L-x_{n}} z_{\sigma(n-1)}^{L-1-x_{n-1}} \ldots z_{\sigma(1)}^{L-n+1-x_{1}} \operatorname{Tr}\left(Q_{n} D_{n}^{L-n} E_{n}^{(\sigma(1))} E_{n}^{(\sigma(2))} \ldots E_{n}^{(\sigma(n))}\right) .
\end{aligned}
$$

We use equation (43) to rearrange the product $E_{n}^{\sigma(1)} \ldots E_{n}^{\sigma(n)}$ in the canonical order $E_{n}^{(1)} \ldots E_{n}^{(n)}$ and obtain

$$
\left\langle x_{1}, \ldots, x_{n} \mid z_{1}, \ldots, z_{n}\right\rangle=K \sum_{\sigma \in \Sigma_{n}}(-1)^{\sigma} \prod_{i=1}^{n}\left(z_{\sigma(i)}-1\right)^{i} z_{\sigma(1)}^{-x_{1}} \ldots z_{\sigma(n)}^{-x_{n}}
$$

with

$$
K=\left(\prod_{i=1}^{n} z_{i}\right)^{L-n} \prod_{i=1}^{n}\left(1-\frac{1}{z_{i}}\right)^{-1} \operatorname{Tr}\left(Q_{n} D_{n}^{L-n} E_{n}^{(1)} E_{n}^{(2)} \ldots E_{n}^{(n)}\right),
$$

and where $(-1)^{\sigma}$ represents the signature of the permutation $\sigma$. We thus find that the eigenvector can be written (Golinelli and Mallick 2005) as a determinant of a matrix $V_{i, j}$ :

$$
\left\langle x_{1}, \ldots, x_{n} \mid z_{1}, \ldots, z_{n}\right\rangle=K \operatorname{det}\left(V_{i, j}\right) \quad \text { with } \quad V_{i, j}=\left(z_{j}-1\right)^{i} z_{j}^{-x_{i}} .
$$

We now show that for the vector (40) to be an eigenvector of the Markov matrix $M$, the pseudo-moments $z_{1}, \ldots z_{n}$ must satisfy the Bethe equations (20). Using the fact that an eigenvector of $M$ is also an eigenvector of the transfer matrix $t(\lambda)$ for any value of $\lambda$ and therefore of the translation operator $\mathcal{T}=t(0)$, we obtain

$$
\begin{aligned}
\left\langle x_{1}, \ldots, x_{n}|\mathcal{T}| z_{1}, z_{2} \ldots z_{n}\right\rangle & =\left\langle x_{1}-1, \ldots, x_{n}-1 \mid z_{1}, z_{2} \ldots z_{n}\right\rangle \\
& =\zeta\left\langle x_{1}, \ldots, x_{n} \mid z_{1}, z_{2} \ldots z_{n}\right\rangle
\end{aligned}
$$

with $\zeta^{L}=1$. We now substitute equation (40) in this identity. We have to distinguish two cases: $x_{1}>1$ and $x_{1}=1$. For $x_{1}>1$, we have

$$
\begin{array}{r}
\operatorname{Tr}\left(Q_{n} D_{n}^{x_{1}-2} E_{n} D_{n}^{x_{2}-x_{1}-1} E_{n} \ldots D_{n}^{x_{n}-x_{n-1}-1} E_{n} D_{n}^{L-x_{n}} D\right)= \\
\zeta \operatorname{Tr}\left(Q_{n} D_{n}^{x_{1}-1} E_{n} D_{n}^{x_{2}-x_{1}-1} E_{n} \ldots D_{n}^{x_{n}-x_{n-1}-1} E_{n} D_{n}^{L-x_{n}}\right)= \\
\left(\prod_{i=1}^{n} z_{i}\right) \operatorname{Tr}\left(Q_{n} D_{n}^{x_{1}-1} E_{n} D_{n}^{x_{2}-x_{1}-1} E_{n} \ldots D_{n}^{x_{n}-x_{n-1}-1} E_{n} D_{n}^{L-x_{n}}\right) .
\end{array}
$$

To derive the last equality, we have used equation (58). We thus have

$$
z_{1} \ldots z_{n}=\zeta
$$


For $x_{1}=1$, equation (69) becomes

$$
\begin{gathered}
\operatorname{Tr}\left(Q_{n} D_{n}^{x_{2}-2} E_{n} \ldots D_{n}^{x_{n}-x_{n-1}-1} E_{n} D_{n}^{L-x_{n}} E_{n}\right)= \\
\zeta \operatorname{Tr}\left(Q_{n} E_{n} D_{n}^{x_{2}-2} E_{n} \ldots D_{n}^{x_{n}-x_{n-1}-1} E_{n} D_{n}^{L-x_{n}}\right) .
\end{gathered}
$$

Using the decomposition (64), we obtain the following sufficient condition for equation (172) to be satisfied by any $\sigma$ :

$$
\begin{array}{r}
\operatorname{Tr}\left(Q_{n} D_{n}^{x_{2}-2} E_{n}^{(\sigma(2))} \ldots D_{n}^{x_{n}-x_{n-1}-1} E_{n} D_{n}^{L-x_{n}} E_{n}^{(\sigma(1))}\right)= \\
\zeta \operatorname{Tr}\left(Q_{n} E_{n}^{(\sigma(1))} D_{n}^{x_{2}-2} E_{n}^{(\sigma(2))} \ldots D_{n}^{x_{n}-x_{n-1}-1} E_{n}^{(\sigma(n))} D_{n}^{L-x_{n}}\right) .
\end{array}
$$

Using equation (43), we commute the operator $E_{n}^{(\sigma(1))}$ with all the other operators $E_{n}^{(\sigma(j))}$, for $j \neq 1$, and bring it back to the rightmost position. This leads to the consistency condition

$$
1=\zeta z_{\sigma(1)}^{L}(-1)^{n-1} \prod_{i=1}^{n} \frac{z_{i}-1}{z_{i}\left(z_{\sigma(1)}-1\right)}
$$

From relation (71), we conclude that this equation is identical to the Bethe equation (20).

\section{References}

- Alcaraz F. C., Droz M., Henkel M., Rittenberg V., 1994, Reactiondiffusion processes, critical dynamics, and quantum chains, Ann. Phys. 230, 250 .

- Alcaraz F. C. and Lazo M. J. , 2004, The Bethe ansatz as a matrix product ansatz, J. Phys. A: Math. Gen. 37, L1; Exact solutions of exactly integrable quantum chains by a matrix product ansatz, J. Phys. A: Math. Gen. 37, 4149.

- Derrida B., 1998, An exactly soluble non-equilibrium system: the asymmetric simple exclusion process, Phys. Rep. 301, 65.

- Derrida B., Evans M. R., Hakim V., Pasquier V., 1993, Exact solution of a $1 D$ asymmetric exclusion model using a matrix formulation, J. Phys. A: Math. Gen. 26, 1493.

- Derrida B. and Lebowitz J. L., 1998, Exact large deviation function in the asymmetric exclusion process, Phys. Rev. Lett. 80, 209. 
- Derrida B., Lebowitz J. L., Speer E. R., 2003, Exact large deviation functional of a stationary open driven diffusive system: the asymmetric exclusion process, J. Stat. Phys. 110, 775.

- Dhar D., 1987, An exactly solved model for interfacial growth, Phase Transitions 9, 51 .

- Golinelli O. and Mallick K., 2004, Hidden symmetries in the asymmetric exclusion process, J. Stat. Mech. P12001.

- Golinelli O. and Mallick K., 2005, Spectral gap of the totally asymmetric exclusion process at arbitrary filling, J. Phys. A: Math. Gen. 38, 1419.

- Gwa L.-H., Spohn H., 1992, Bethe solution for the dynamical-scaling exponent of the noisy Burgers equation, Phys. Rev. A 46, 844.

- Kim D., 1995, Bethe ansatz solution for crossover scaling functions of the asymmetric XXZ chain and the Kardar-Parisi-Zhang-type growth model, Phys. Rev. E 52, 3512.

- Mallick K., 1996, Systèmes hors d'équilibre: quelques résultats exacts, PhD Thesis, University of Paris 6.

- Nepomechie R.I., 1999, A Spin Chain Primer, Int. J. Mod. Phys. B 13, 2973 (hep-th/9810032).

- Schütz G. M., 2001, Phase Transitions and Critical Phenomena, Vol. 19, C. Domb and J. L. Lebowitz Eds, (Academic, London).

- Speer E. R., 1993, The two species totally asymmetric exclusion process, in Micro, Meso and Macroscopic approaches in Physics, M. Fannes et al. Eds. (NATO Workshop, Leuven, 1993).

- Stinchcombe R. B. and Schütz G. M., 1995, Application of operator algebras to stochastic dynamics and the Heisenberg chain, Phys. Rev. Lett. 75, 140. 